temporal lobectomy for intractable epilepsy. Neurology 1998; 51:465-471.

14. Ho SS, Consalvo D, Gilliam F, et al. Amygdala atrophy and seizure outcome after temporal lobe epilepsy surgery. Neurology 1998;51:1502-1504.

15. Li LM, Cendes F, Antel SB, et al. Prognostic value of proton magnetic resonance spectroscopic imaging for surgical outcome in patients with intractable temporal lobe epilepsy and bilateral hippocampal atrophy. Ann Neurol 2000;47:195-200.

16. Ende GR, Laxer KD, Knowlton RC, et al. Temporal lobe epilepsy: bilateral hippocampal metabolite changes revealed at proton MR spectroscopic imaging. Radiology 1997;202:809817.

17. Grigsby J, Kramer RE, Schneiders JL, Gates JR, Smith WB. Predicting outcome of anterior temporal lobectomy using simulated neural networks. Epilepsia 1998;39:61-66.

18. Arle JE, Perrine K, Devinsky O, Doyle WK. Neural network analysis of preoperative variables and outcome in epilepsy surgery. J Neurosurg 1999;90:998-1004.

19. Duda RO, Hart PE. Pattern classification and scene analysis. New York: Wiley, 1973.

20. Mitchell TM. Machine learning. Boston: WCB McGraw-Hill, 1997.

21. Engel JJ, Van Ness PC, Rasmussen TB, Ojemann LM. Outcome with respect to epileptic seizures. In: Engel JJ, ed. Surgical treatment of the epilepsies. 2nd ed. New York: Raven Press, 1993:609-621.
22. Watson C, Andermann F, Gloor P, et al. Anatomic basis of amygdaloid and hippocampal volume measurement by magnetic resonance imaging. Neurology 1992;42:1743-1750.

23. Roth K, Kimber BJ, Feeney J. Data shift accumulation and alternate delay accumulation techniques for overcoming the dynamic range problem. J Magn Res 1980;41:302-309.

24. Hand DJ. Discrimination and classification. Chichester: Wiley, 1981.

25. Webb A. Statistical pattern recognition. London: Oxford University Press, 1999.

26. Kuzniecky R, Ho SS, Martin R, et al. Temporal lobe developmental malformations and hippocampal sclerosis: epilepsy surgical outcome. Neurology 1999;52:479-484.

27. Kuzniecky R, Hugg J, Hetherington H, et al. Predictive value of ${ }^{1} \mathrm{H}$ MRSI for outcome in temporal lobectomy. Neurology 1999;53:694-698.

28. Jack CR Jr, Trenerry MR, Cascino GD, Sharbrough FW, So EL, O'Brien PC. Bilaterally symmetric hippocampi and surgical outcome. Neurology 1995;45:1353-1358.

29. Siegel AM, Wieser HG, Wichmann W, Yasargil GM. Relationships between MR-imaged total amount of tissue removed, resection scores of specific mediobasal limbic subcompartments and clinical outcome following selective amygdalohippocampectomy. Epilepsy Res 1990;6:56-65.

30. Wyler AR, Hermann BP, Somes G. Extent of medial temporal resection on outcome from anterior temporal lobectomy: a randomized prospective study. Neurosurgery 1995;37:982-991.

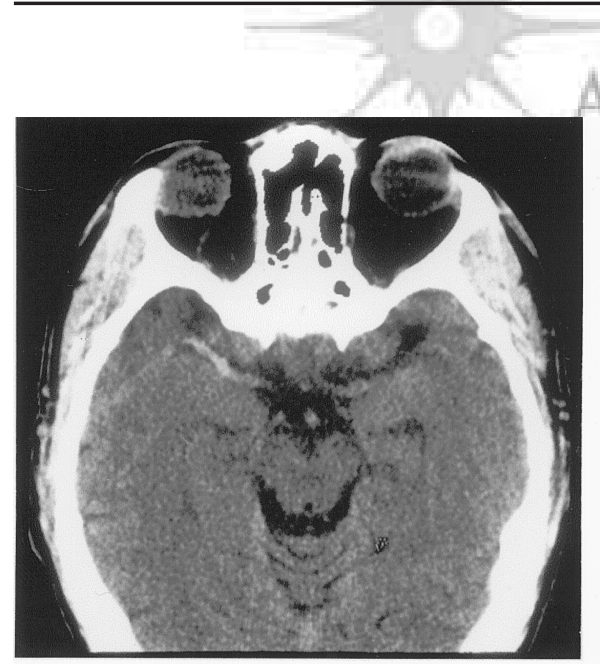

A

\section{Hyperdense MCA resolved after tPA}

J.W. Tsao, MD, DPhil, A.D. Gean, MD, O.A. Glenn, MD, D.C. Bonovich, $M D$, San Francisco, $C A$

A 57-year-old male experienced sudden onset of left-sided hemianopsia, neglect, and weakness (face/arm [0/5 strength] $>$ leg [2/5 strength]); NIH stroke scale (NIHSS) $=16$. Prothrombin time/international normalized ratio was 15.2/1.8. CT scan revealed a hyperdense middle cerebral artery (HMCA) (figure, A). tPA $(0.9 \mathrm{mg} / \mathrm{kg})$ was infused at $\mathrm{t}=2.5$ hours. Within 30 minutes, arm/leg strength was 4/5 (NIHSS 10). Repeat CT scan demonstrated MCA patency (see figure, B). The patient made a complete recovery.

The HMCA is a marker of intraluminal thrombus in the appropriate clinical setting. ${ }^{1}$ When accompanied by an NIHSS $>10$, outcomes are typically poor. ${ }^{2}$ We believe these images represent recanalization of an occluded vessel.

1. Gacs G, Fox AJ, Barnett HJM, et al. CT visualization of intracranial arterial thromboembolism. Stroke 1983;14:756-762.

2. Tomsick T, Brott T, Barsan W, et al. Prognostic value of the hyperdense middle cerebral artery sign and stroke scale score before ultraearly thrombolytic therapy. AJNR Am J Neuroradiol 1996;17:79-85. 


\section{Neurology}

\section{Hyperdense MCA resolved after tPA}

J. W. Tsao, A. D. Gean, O. A. Glenn, et al.

Neurology 2002;58;1512

DOI 10.1212/WNL.58.10.1512

\section{This information is current as of May 28, 2002}

\section{Updated Information \& Services}

\section{References}

Citations

Subspecialty Collections

Permissions \& Licensing

Reprints including high resolution figures, can be found at: http://n.neurology.org/content/58/10/1512.full

This article cites 2 articles, 1 of which you can access for free at: http://n.neurology.org/content/58/10/1512.full\#ref-list-1

This article has been cited by 1 HighWire-hosted articles: http://n.neurology.org/content/58/10/1512.full\#\#otherarticles

This article, along with others on similar topics, appears in the following collection(s):

Embolism

http://n.neurology.org/cgi/collection/embolism

Infarction

http://n.neurology.org/cgi/collection/infarction

MRI

http://n.neurology.org/cgi/collection/mri

Information about reproducing this article in parts (figures,tables) or in its entirety can be found online at:

http://www.neurology.org/about/about_the_journal\#permissions

Information about ordering reprints can be found online:

http://n.neurology.org/subscribers/advertise

Neurology ${ }^{\circledR}$ is the official journal of the American Academy of Neurology. Published continuously since 1951, it is now a weekly with 48 issues per year. Copyright . All rights reserved. Print ISSN: 0028-3878.

Online ISSN: 1526-632X.

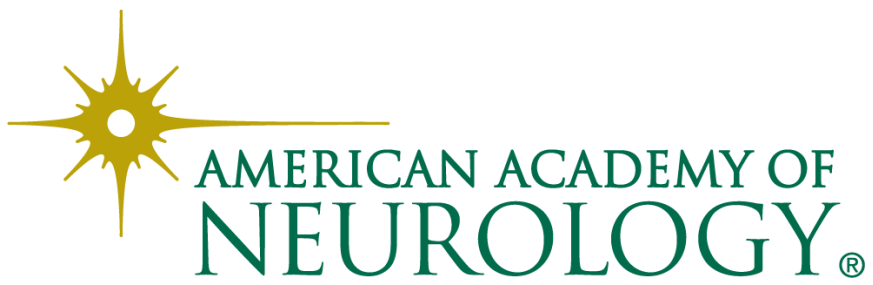

